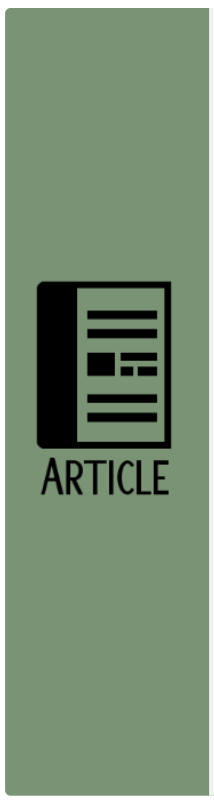

\title{
Comprendre et agir : Diagnostic agronomique et modélisation d'accompagnement pour limiter le risque d'érosion dans un bassin versant montagnard subtropical
}

Trébuil Guy*1, Barnaud Cécile², Bousquet François ${ }^{3}$, Promburom Panomsak4, Turkelboom Francis 5 , van Paassen Annemarie ${ }^{6}$

${ }^{1}$ CIRAD, UMR Innovation, F-34398 Montpellier, France \& Innovation, Univ. Montpellier, CIRAD, INRAE, Montpellier SupAgro, Montpellier, France

${ }^{2}$ DYNAFOR, Université de Toulouse, INPT, INRAE, Toulouse, France

3 CIRAD, UMR SENS, F-34398 Montpellier, France \&

SENS, Univ. Montpellier, CIRAD, IRD, Univ. Paul Valéry, Montpellier, France

${ }^{4}$ Center of Agricultural Resource System Research, Chiang Mai University, 50200 Thailand

${ }^{5}$ Research Institute for Nature and Forest INBO, Kliniekstraat 25, 1070Brussels, Belgique

${ }^{6}$ Communication and Innovation, Wageningen University and Research Center, Pays-Bas

*Auteur correspondant : guy.trebuil@gmail.com

\section{Résumé}

Une enquête-diagnostic agronomique multi-niveaux permit de comprendre la dynamique du risque d'érosion des sols dans un bassin versant soumis à une diversification rapide des cultures et exploitations familiales. Les résultats intégrés dans un modèle multi-agent de chercheurs représentaient les impacts de la topographie, de la pluviométrie et des actes techniques sur le risque d'érosion. Il fut simplifié et soumis aux acteurs locaux pour validation sous la forme d'un jeu de rôles et de son modèle de simulation informatique associé. Ces outils permirent d'initier une modélisation d'accompagnement (ComMod) pour partager des points de vue, construire un diagnostic partagé et stimuler l'action collective afin de limiter l'érosion et la vulnérabilité des exploitations défavorisées. Les séquences successives d'activités ComMod conduisaient à compléter le diagnostic agronomique initial au fil des questions clés débattues par les agriculteurs. Elles entraînèrent les acteurs locaux dans des directions jusque-là non proposées par les organismes de développement agricole et produisirent de multiples effets en matière de prise de conscience, confiance en soi, communication, apprentissage, changements de pratiques et autoorganisation. L'institutionnalisation de cette démarche permettrait un accompagnement plus suivi, faciliterait la diffusion des résultats, ainsi que l'évolution de la gouvernance locale des ressources renouvelables.

Mots-clés: Enquête agronomique, modèle multi-agent, jeu de rôles, simulation participative, Thaïlande

\section{Abstract Understand and act: Agronomic diagnosis and companion modelling to limit soil erosion risk in a subtropical mountain catchment}

An on-farm agronomic survey was carried out, at the field, farm and catchment levels, to understand the dynamics of the risk of soil erosion in a watershed displaying a rapid diversification of cropping systems and differentiation of family farms. The results were integrated into a researcher agent-based model linked to a geographic information system to represent dynamically the combined impacts of topography, rainfall and technical practices on erosion risk and its spatial distribution. A simpler version of this model was implemented as a role-playing game associated to 
a second agent-based model playing the game in silico, to share the researchers' point of view on soil erosion dynamics to local farmers. They found these simulation tools relevant and Companion Modelling (ComMod) activities where launched with them to stimulate exchanges of experiences, build a shared diagnosis of the problem, and encourage collective action to limit soil degradation and the vulnerability of resource-poor holdings. The successive cycles of ComMod activities necessitated to complete the initial agronomic diagnosis by examining a series of farmer selected key questions. Gradually, local stakeholders moved towards directions not yet proposed by development agencies. The activities produced multiple effects related to awareness, selfconfidence and communication, learning, change in practices and self-organization. The limited durations of successive training projects were not adapted to accompany local actors up to collective action at the catchment level, but new irrigation water and farm product processing infrastructures emerged a few years later in the village. The institutionalization of the approach would allow a more continuous companionship, facilitate the dissemination of results, and a move towards a more distributed governance of renewable resources.

Keywords: Agronomic survey, multi-agent model, role-playing game, participatory simulation, Thailand

\section{Introduction}

L'importance du problème de dégradation des terres cultivées à l'échelle mondiale est à nouveau soulignée à l'heure de l'accélération du changement climatique et notamment l'augmentation de la fréquence et de l'intensité des évènements pluvieux extrêmes en de nombreux endroits. Depuis les années 80 , les transformations rapides des systèmes montagnards d'Asie du Sud-Est continentale, de l'abattis-brûlis à longues jachères non labourées et fort niveau d'autosubsistance vers des systèmes de culture continue marchands et diversifiés alimentant une agro-industrie florissante (Trébuil et al., 2006), ont conduit à s'interroger quant à l'évolution du risque d'érosion des sols. Cette diversification commerciale accentuant par ailleurs les inégalités entre les exploitations familiales des hautes terres, il s'agissait de tester des manières adaptées de limiter le risque de dégradation des terres tout en limitant la vulnérabilité des exploitations familiales de ces agroécosystèmes surplombant les vastes « bols de riz » irrigués de pays exportateurs majeurs.

L'agriculture de petite montagne du « triangle d'or » à l'extrême nord de la Thaïlande a constitué un laboratoire de la recherche-développement internationale sur la lutte contre l'érosion. Mais au début des années 90, l'échec de dizaines de projets reposant sur de vaines tentatives de normalisation de «bonnes pratiques » importées (bandes enherbées et haies antiérosives en courbes de niveau avec espèces variées, mise en terrasses des versants, etc.) était patent. Leurs recommandations techniques prescriptives descendantes, déconnectées de la diversité des situations paysannes locales, affichaient des taux d'adoption minimes, malgré les onéreux moyens déployés, dans cette région de la «Zomia » où l'on cultive de longue date "l'art de ne pas être gouverné » (Scott, 2013) et privilégie l'auto-organisation. En l'absence de réduction significative de la dégradation des terres, les populations minoritaires montagnardes demeuraient souvent menacées d'éviction par les autorités nationales.

Une approche innovante fut testée pour à la fois comprendre les dynamiques agronomiques à l'œuvre grâce à l'analyse-diagnostic de situations paysannes variées, et utiliser ses résultats afin de concevoir de nouveaux usages des terres avec les acteurs locaux, ainsi que les mobiliser dans l'action collective au niveau territorial.

Au tournant des années 2000, les postures du diagnostic-conseil de l'agronome en milieu paysan et de pilotage externe de ces agroécosystèmes montagnards dominaient. Cependant, l'urgence des problèmes de dégradation des ressources renouvelables et la montée des approches participatives suscitaient une demande d'agronomes-médiateurs pour, en lien avec les sciences sociales, faciliter le dialogue et la coordination entre un nombre croissant de catégories d'acteurs dans les bassins versants. Les difficultés de mise en œuvre de ce type d'approche sont connues : 
comment articuler les différents niveaux d'organisation pertinents et représenter les dynamiques agronomiques et sociales en interaction? Comment intégrer leurs fortes incertitudes et la rapidité du changement? Comment dépasser les intérêts individuels contrastés et faire émerger des mécanismes de coordination facilitant l'action collective concertée?

L'approche choisie s'appuyait sur une analyse-diagnostic approfondie du fonctionnement de l'agroécosystème (de la station intra-parcellaire à pente homogène, au champ cultivé, à l'exploitation familiale et au bassin versant, 1994-98). Ses résultats furent représentés dans un modèle d'accompagnement de la réflexion des chercheurs (1999-2000), dont la restitution aux acteurs locaux permit d'amorcer une modélisation d'accompagnement (ComMod) (Bousquet, 2001 ; Etienne coord., 2010) de leur projet pour le territoire (2001-2007). Cette approche a été mise en œuvre dans deux villages (Pakha et Mae Salaep) de l'ethnie Akha de la province de Chiang Rai, assez isolés à l'extrême nord de la Thaïlande et incomplètement intégrés dans la société thaïe. Cette approche innovante pouvait-elle fonctionner dans une situation agraire aussi (agronomiquement, économiquement, socio-culturellement et institutionnellement) difficile?

L'article montre comment les résultats de l'enquête agronomique pour comprendre et représenter les interactions entre la diversification des systèmes de culture et le risque d'érosion ont facilité l'initiation d'une modélisation d'accompagnement. Cette dernière devait renforcer le partage de connaissances, identifier des voies adaptées d'amélioration des pratiques, explorer et évaluer collectivement des scénarios d'évolution de l'usage des terres et conduire à des actions collectives sur le territoire. Entre chaque séquence d'activités ComMod focalisée sur une question-clé mise en débat, le diagnostic agronomique était complété et actualisé à propos des nouveaux sous-systèmes à prendre en compte. Nous montrons comment ces activités itératives et évolutives ont entraîné les acteurs locaux dans une direction très différente de celles prônées jusque-là par le conseil technique et le pilotage extérieur de ces agroécosystèmes.

Les cinq phases successives de cette intervention, leur articulation et principaux résultats sont brièvement présentés. La description des effets observés et l'évaluation des activités repose ensuite sur une analyse réflexive $a$ posteriori des enseignements de cette expérimentation. Elle identifie aussi les points forts et les faiblesses de l'approche et des outils mobilisés et traite des perspectives d'amélioration en conclusion.

\section{Une recherche-action impliquée en cinq phases}

\section{L’enquête-diagnostic agronomique multi-échelles et ses principaux résultats}

En mobilisant la diversité des situations culturales des champs cultivés (angles et longueurs de pente, successions de cultures, itinéraires techniques), l'enquête-diagnostic se déroula sur 2 saisons des pluies (1994 et 1995) pour comprendre la relation entre risque d'érosion par ruissellement concentré et diversification des systèmes de culture (Turkelboom 1999 ; Turkelboom et al., 1996 et 2008). Inspirée de la méthode de Boiffin et al. (1988) dans le pays de Caux, elle a caractérisé et suivi l'évolution des états de la culture et du milieu après chaque pluie érosive d'au moins $10 \mathrm{~mm}$, susceptible de créer des symptômes visibles, sur 51 petites zones à pente homogène délimitées dans des champs fournissant une gamme étendue de situations culturales contrastées. Les différents types d'érosion à l'œuvre et leurs degrés de sévérité respectifs en fonction de l'angle et la longueur de pente, la couverture du sol et l'histoire culturale de la parcelle furent identifiés, ainsi que les seuils de basculement entre types de processus érosifs présentés dans le tableau 1. L'enquête permit aussi d'identifier la sensibilité des principales cultures au risque d'érosion. La durée de leurs périodes critiques (nombre de jours après semis) était de $121 \pm 28$ j pour le riz pluvial, $44 \pm 13$ pour le maïs, $38 \pm 9$ pour les légumineuses à graines et $38 \pm 6$ j pour le chou commun. 
Tableau 1: Risque d'érosion par ruissellement concentré selon la situation culturale :\% de parcelles enquêtées présentant de nouveaux symptômes d'érosion après une pluie érosive (> $10 \mathrm{~mm}$ ).

\begin{tabular}{|l|l|c|c|c|c|}
\hline \multirow{2}{*}{$\begin{array}{c}\text { Couverture du sol inférieure au } \\
\text { seuil critique de : }\end{array}$} & \multirow{2}{*}{ Histoire culturale } & \multicolumn{3}{|c|}{ Angle et longueur de pente } \\
\cline { 3 - 6 } & & \multicolumn{2}{|c|}{$<57 \%$} & \multicolumn{2}{|c|}{$>57 \%$} \\
\cline { 3 - 6 } & & $<25 \mathrm{~m}$ & $\geq 25 \mathrm{~m}$ & $<25 \mathrm{~m}$ & $\geq 25 \mathrm{~m}$ \\
\hline $\begin{array}{l}<0 \% \text { de couverture totale ou } \\
<30 \% \text { de couverture de contact }\end{array}$ & $1^{\text {ère }}$ année après jachère & $7 \%$ & $7 \%$ & $25 \%$ & $50 \%$ \\
\cline { 2 - 6 } & $\geq 2^{\text {ème }}$ année de culture & $29 \%$ & $29 \%$ & $33 \%$ & $51 \%$ \\
\hline
\end{tabular}

L'enquête quantifia aussi les pertes en terre dues à l'érosion sèche provoquée mécaniquement par le travail du sol à la houe sur pentes raides, et identifia les facteurs responsables de dégradations majeures à l'échelle du petit bassin versant comme les glissements de terrain (Turkelboom, 1999; Turkelboom et al., 2008).

Parallèlement, l'analyse du fonctionnement des 48 petites exploitations familiales du village de Mae Salaep en fonction de leurs stratégies de diversification, du niveau de risque économique accepté, et de la localisation topographique de leurs champs liée à leur histoire, permit d'identifier trois grands types d'exploitations :

- Type A: petites exploitations de jeunes producteurs de cultures commerciales établies récemment, le plus souvent sur de fortes pentes,

- Type B : exploitations de taille moyenne combinant cultures d'autosubsistance et commerciales, mais avec forte aversion au risque de variation des prix,

- Type $C$ : plus grandes exploitations diversifiées et intégrées aux filières horticoles, sur pentes plus faibles par des familles établies de longue date.

L'analyse des exploitations avec visites répétées introduisit l'équipe de recherche chez les agriculteurs et permit de clarifier ses objectifs. Elle facilita l'établissement d'une relation de confiance avant l'organisation des ateliers d'une semaine à suivre, ainsi que l'engagement durable des 12 agriculteurs/trices des 3 types identifiés invités à y participer.

Une cartographie du parcellaire intégrée dans un système d'information géographique (SIG) servit à spatialiser le risque d'érosion dans le principal bassin versant villageois comprenant environ 1500 petites zones à pente homogène dans 220 champs gérés par les 3 types d'exploitations familiales (Turkelboom et Trébuil, 1998).

\section{Intégration des résultats de l'enquête dans un modèle d'accompagnement de chercheurs}

Un modèle dynamique multi-agent, spatialisé et multi-niveaux fut construit pour intégrer les connaissances issues du diagnostic agronomique sur les dynamiques et décisions importantes des agriculteurs à chacune des 4 échelles enquêtées (Trébuil et al., 2005). L'estimation du risque d'érosion s'effectuait à l'échelle de la petite zone à pente homogène, sur la base des seuils du tableau 1, puis était agrégés aux échelles du champ cultivé, du type d'exploitation et du bassin versant. Ce modèle complexe d'accompagnement des chercheurs dans leur compréhension des dynamiques permettait de simuler la distribution spatiale du risque érosif selon les assolements des différents types d'exploitations et la pluviométrie aux pas de temps quotidien ou du cycle cultural. Il permettait aussi de représenter l'influence des itinéraires techniques sur l'érosion au pas de temps quotidien en périodes critiques, notamment l'effet des dates de travail du sol et de sarclage en fonction de celles des pluies les plus intenses. La grande majorité des pertes en terres étaient occasionnées chaque année par un petit nombre de pluies intenses, comme indiqué sur la figure 1. 


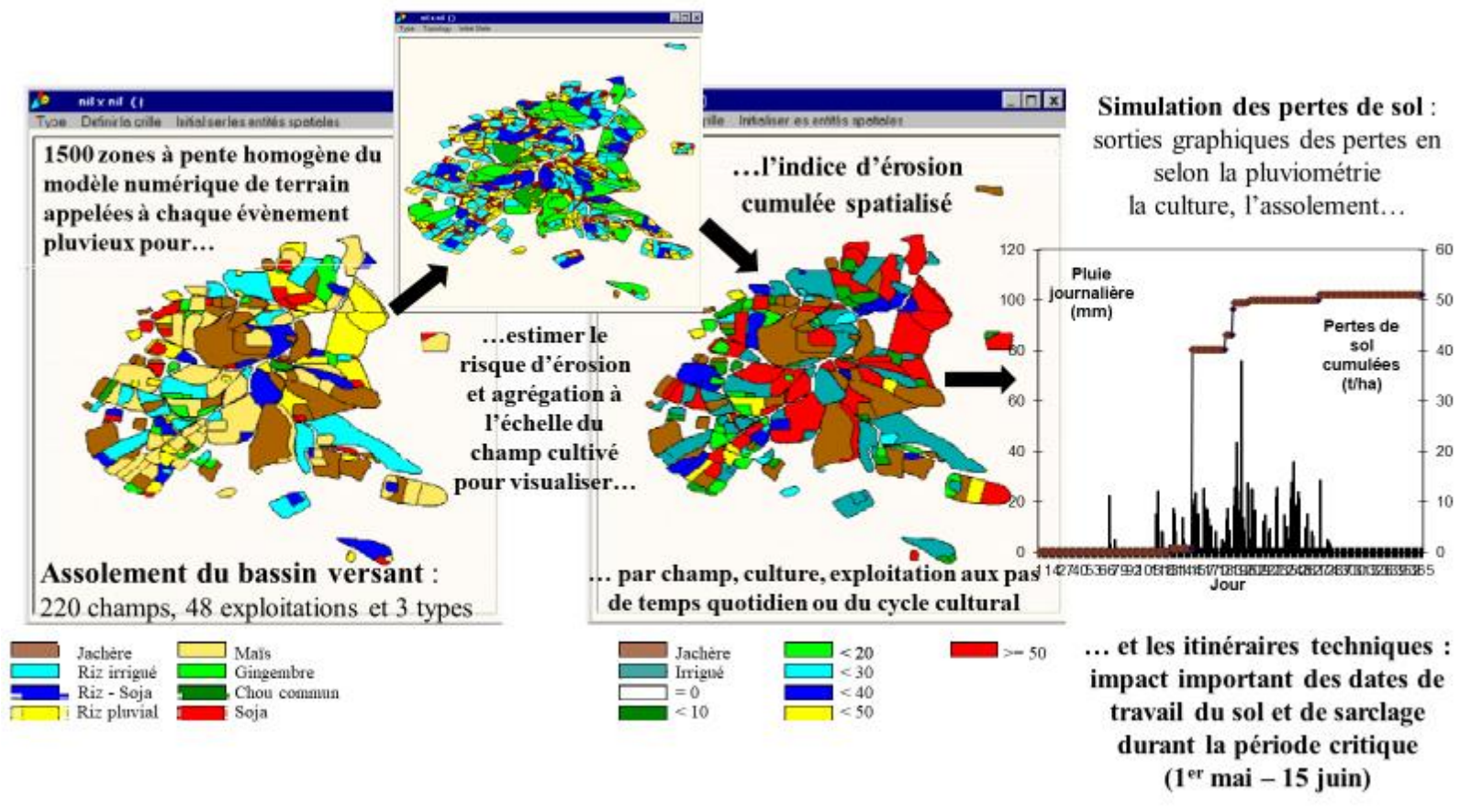

Figure 1 : Intégration des connaissances du diagnostic agronomique dans un modèle de simulation multiagent - SIG multi-niveaux pour visualiser et spatialiser le risque d'érosion.

\section{Restitution des résultats du diagnostic et initiation des activités ComMod}

Afin de faciliter sa compréhension par les agriculteurs et le vulgarisateur agricole local, ainsi que pour leur restituer les résultats de l'enquête-diagnostic, ce modèle multi-agent couplé à un SIG (SMA-SIG) représentant les connaissances acquises (voir Trébuil et al., 2005 pour une description détaillée) fut simplifié, puis présenté sous sa forme initiale seulement dans un second temps.

La version simplifiée était un modèle multi-agent dans lequel seules les interactions majeures étaient prises en compte : 4 types de pentes, 3 niveaux de pluviométrie, les itinéraires techniques les plus fréquents de 3 grands types de cultures, et 3 types d'exploitations. Ce modèle SMA simplifié fut implémenté en un premier jeu de rôles $(J d R)$ utilisé durant un atelier de quelques jours au village. 12 femmes et hommes gérant différents types d'exploitations familiales y participèrent, l'institutrice Akha trilingue jouant un rôle clef dans son animation.

Chaque joueur gérait le même type d'exploitation que dans la réalité en choisissant des cultures pour chacune de ses parcelles plus ou moins pentues, et observait en fin de tour de jeu l'ampleur des symptômes d'érosion créés en fonction d'une pluviométrie tirée au hasard après les semis. Après les séances de $J d R$, sa version informatique jouant le jeu in silico fut introduite pour réaliser des simulations participatives. Les participants, dont plusieurs n'avaient reçu aucune éducation formelle, ne montrèrent guère de difficultés à se l'approprier (Figure 2). Le modèle de chercheur SMA-SIG complet était ensuite présenté, mais ses visualisations réalistes amenaient les agriculteurs à se focaliser sur les dynamiques de leurs parcelles, perdant ainsi une vue plus large des résultats des simulations.

Une première association entre les outils $J d R$ et modèle informatique multi-agent (SMA) jouant le jeu était donc proposée à ce stade. Ces 2 outils de simulation étant au cœur des activités d'une séquence ComMod comme indiqué sur la figure 3. 


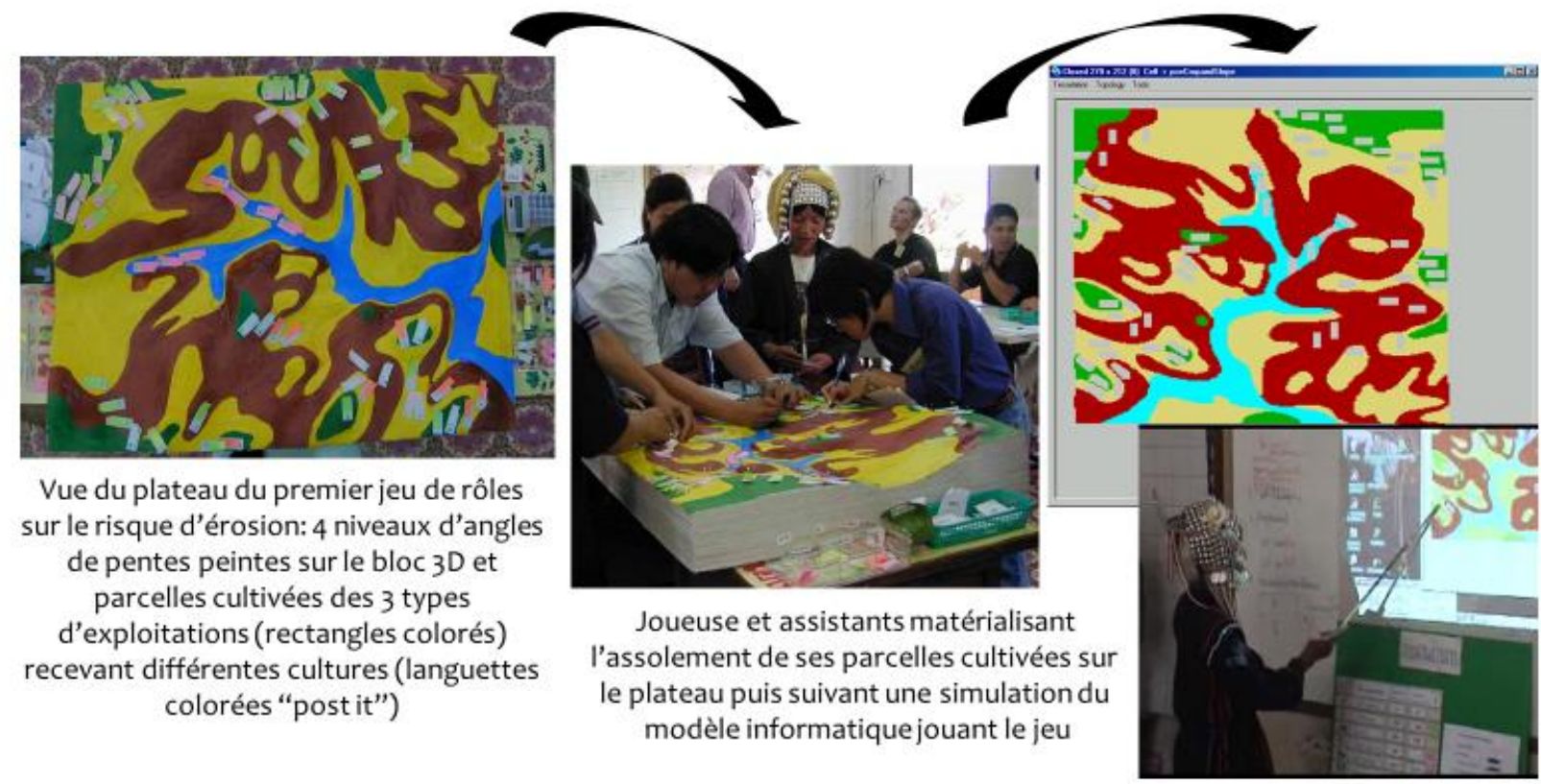

Figure 2 : Modèle simplifié implémenté en un jeu de rôles associé à un simulateur informatique multi-agents jouant le jeu pour explorer les scénarios sélectionnés par les participants aux ateliers

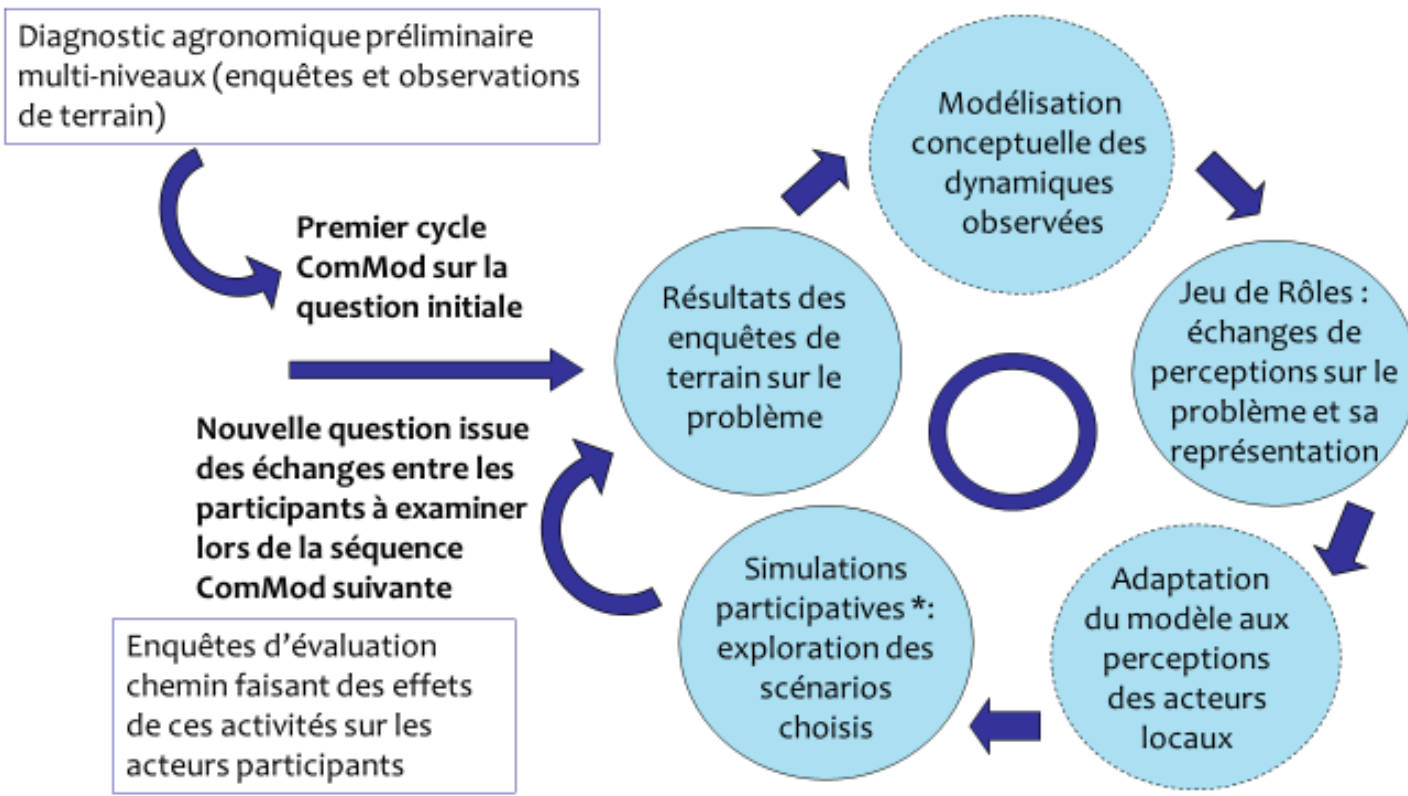

* A I'aide d'un simulateur informatique Multi-Agents et/ou d'un Jeu de rôles

Figure 3: Principales phases d'une séquence d'activités de modélisation d'accompagnement

Les résultats du diagnostic agronomique ainsi soumis aux agriculteurs, les participants à l'atelier les jugèrent réalistes lors du débriefing final. Cependant, menacés d'éviction sous la pression d'une politique nationale de reforestation des hauts de bassins versants, ils déclaraient que « cela montre bien le problème, mais il nous faut vite trouver des solutions ». Pour accroître le taux de couverture du sol lors des périodes critiques, plutôt que de changer leurs pratiques en cultures annuelles, ils souhaitaient explorer la possibilité d'étendre les cultures commerciales pérennes (thé vert et litchis notamment), mais pointaient l'accès limité au crédit pour investir dans ces plantations. La mise en 
œuvre de la séquence suivante d'activités ComMod porta sur ce thème. Comme les animateurs demandaient aux participants de sélectionner le type de modèle (SMA-SIG initial ou simplifié) à utiliser pour représenter les sous-systèmes pertinents et simuler les scénarios les plus intéressants, les agriculteurs choisissaient le SMA simplifié associé à un nouveau JdR.

\section{$2^{\text {ème }}$ séquence d'activités ComMod : expansion des cultures pérennes et accès au crédit rural}

En accord avec les préoccupations des agriculteurs, cette séquence était dominée par des dynamiques économiques (accès au crédit formel et informel selon le type d'exploitation, main d'œuvre disponible et opportunités d'emplois hors exploitation, rentabilité des culture pérennes et durabilité des petites exploitations) et sociales (réseaux d'accointances plus ou moins étendus selon les exploitations pour l'accès au crédit) (Barnaud et al., 2007). Ceci montre l'intérêt d'une approche transdisciplinaire à l'interface entre dynamiques agronomiques et socio-économiques afin de pouvoir examiner les questions et thématiques qui émergent des échanges entre acteurs. La composante agronomique consistait à analyser les pratiques en productions pérennes préférées des agriculteurs : le thé vert pluvial de type Assam chez les exploitations A et B (le prix d'achat du thé variant peu) et le litchi préféré par les agriculteurs mieux dotés en facteurs de production et pouvant faire face à une très forte fluctuation du prix d'achat.

Le modèle conceptuel simplifié précédent était adapté au nouveau thème en tenant compte de la diversification des sources de crédit accessibles et cette nouvelle version du modèle était implémentée en un nouveau couple $J d R$ (pour paramétrer et valider la représentation des dynamiques avec les acteurs locaux) associé au simulateur informatique multi-agent jouant le jeu beaucoup plus rapidement et permettant ainsi l'exploration de scénarios simulés sur quelques années (Barnaud et al., 2008a). Le fait que les acteurs locaux acceptèrent de faire figurer explicitement dans le modèle leur façon de contourner les règles d'attribution du crédit formel montrait que l'outil de simulation était pris au sérieux. L'évaluation collective des scénarios reposait sur des indicateurs agronomiques (surfaces en cultures pérennes selon le type d'exploitation) et socio-économiques (taux d'élimination des exploitations endettées) intéressant les participants. Les simulations, réalisées avec les mêmes participants de la séquence précédente mettaient en évidence l'inégalité d'accès au crédit formel selon le type d'exploitation et l'importance du crédit informel (auprès de parents et membres du clan) pour limiter le risque de faillite des plus petites exploitations. Le crédit formel semblait plus efficace pour accroître la superficie des plantations, à condition que la période de remboursement soit allongée et une requête collective pour aller dans ce sens fut adressée aux autorités provinciales. Grâce au crédit informel, un réseau d'accointances élargi était un moyen privilégié pour les exploitations les plus fragiles d'éviter le recours aux usuriers et la banqueroute en cas de baisse des prix.

Cette séquence illustrait le compromis à réaliser entre favoriser la dynamique d'expansion des plantations et limiter les inégalités sociales entre agriculteurs. Ce dilemme allait s'approfondir lors de la séquence suivante qui remettait les dynamiques agronomiques au premier plan. Les cultures de thé Oolong et de litchi ayant recours à l'irrigation, les participants demandèrent qu'elle portât sur la gestion collective de l'eau dans le bassin versant pour stabiliser la qualité des produits horticoles et les revenus. Plus confiants dans leur capacité à élaborer un projet d'aménagement, ils demandèrent aussi d'impliquer les élus de l'organisation administrative du sous-district qui disposaient de financements pour soutenir un tel projet. Avec le bassin versant comme principal niveau d'organisation considéré et l'accroissement de l'hétérogénéité des acteurs locaux impliqués, les activités de cette $3^{\text {ème }}$ séquence pouvaient contribuer à la construction ascendante d'un projet de développement territorial (Figure 4). 


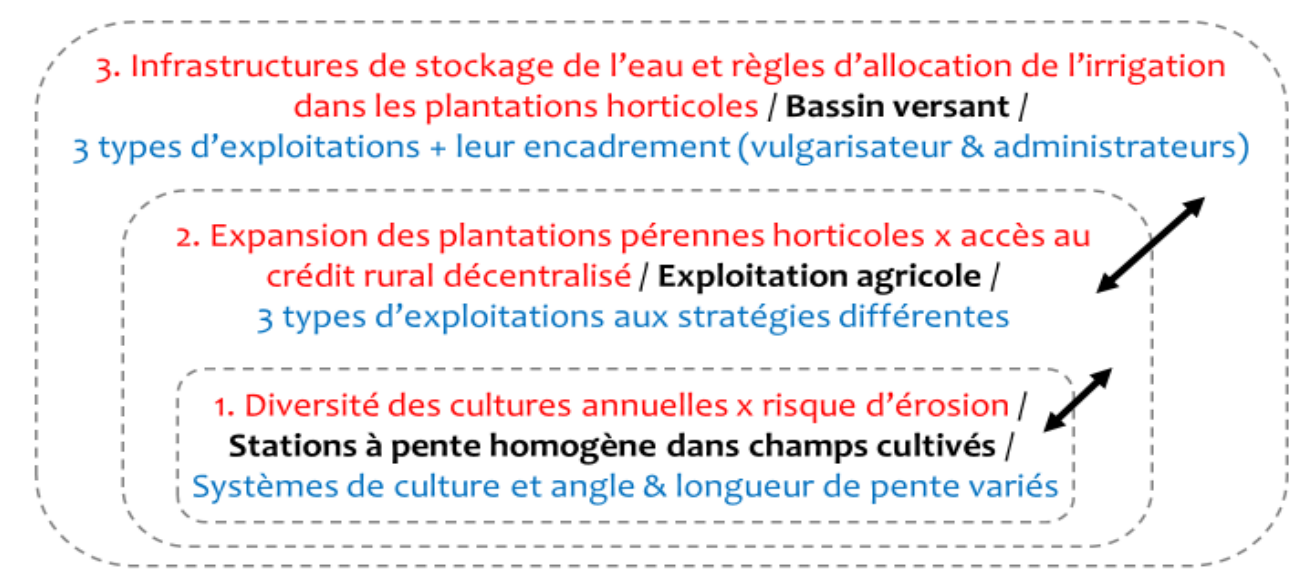

Légende : focus de la séquence / Niveau d’organisation / Hétérogénéité prise en compte

Figure 4 : Évolution de la question clé, du niveau d'organisation du territoire et des participants impliqués dans les trois séquences successives de modélisation d’accompagnement à Mae Salaep

\section{$3^{\text {ème }}$ séquence d'activités ComMod sur la gestion collective de l'irrigation}

La création de nouvelles infrastructures de stockage de l'eau était jugée nécessaire pour augmenter le volume disponible face à l'expansion des plantations horticoles, mais aussi afin de pouvoir changer les règles inéquitables de distribution de la ressource en vigueur. Une courte enquête-diagnostic sur les pratiques d'irrigation montrait que la règle du premier arrivé premier servi favorisait les plus grandes exploitations établies de longue date, tandis qu'une majorité d'agriculteurs n'y avaient pas accès. Or l'introduction récente de la culture du thé vert Oolong, de qualité supérieure et plus rémunérateur, depuis un district voisin nécessitait le recours à l'irrigation. Le modèle conceptuel était à nouveau adapté à la nouvelle thématique et implémenté en un zème couple JdR - simulateur informatique multi-agent pour l'exploration de scénarios sur le partage de l'eau agricole (Barnaud et al., 2008b). Les indicateurs choisis par les usagers étaient respectivement la répartition entre exploitations du volume d'eau disponible et le revenu brut des plantations des exploitations se partageant la ressource d'un même cours d'eau (cf. le graphique de la figure 5). Tandis que les plus grandes exploitations horticoles d'un clan influent du village, représenté par un élu de l'administration du sous-district, plaidaient pour la construction d'une retenue unique alimentant seulement une partie du bassin, les autres agriculteurs défendaient la construction de petites retenues collinaires desservant les groupes d'exploitations situées le long de chacune des rivières. Si les exploitants aisés défendaient l'attribution de volumes d'eau d'irrigation proportionnels à la superficie des plantations, l'autre groupe (majoritaire) souhaitait que chaque exploitation puisse disposer d'un volume avec possibilité d'en céder aux grandes exploitations, ceci afin que tous les ménages puissent bénéficier des aménagements réalisés, notamment ceux cultivant des parcelles hautes non irrigables. La figure 5 montre que chaque groupe testa son scénario préféré séparément afin de permettre une expression équitable des points de vue, mais le débriefing en séance plénière ne permit pas de réconcilier les deux visions. L'influent leader des exploitants aisés plaidait pour soumettre son projet à l'administration locale mais était systématiquement contré par les autres types d'exploitants. Ce groupe des plus petites exploitations $A$ et $B$ gagnait en cohésion et, rassemblé autour de leur leader religieux charismatique, décidait après l'atelier de mettre en œuvre leur projet avec l'aide d'autres sources de financement et du lycée technique professionnel local (Barnaud et al., 2010).

Lors du débriefing final de ce $3^{\text {ème }}$ atelier ComMod, les participants demandaient aussi à ce qu'une 4ème séquence d'activités similaires soit organisée sur le thème du conditionnement local et de la commercialisation du thé vert afin de maximiser la part de la plus-value revenant au village. Le projet arrivant à son terme alors que la démarche était encore insuffisamment institutionnalisée et dépendante de doctorants en formation, cette nouvelle séquence ne put avoir lieu. 


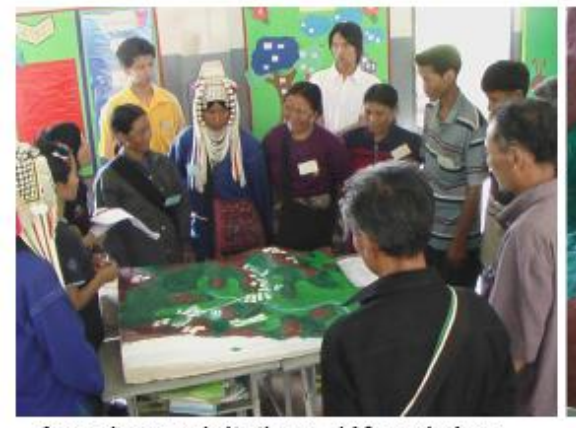

Avec les exploitations défavorisées
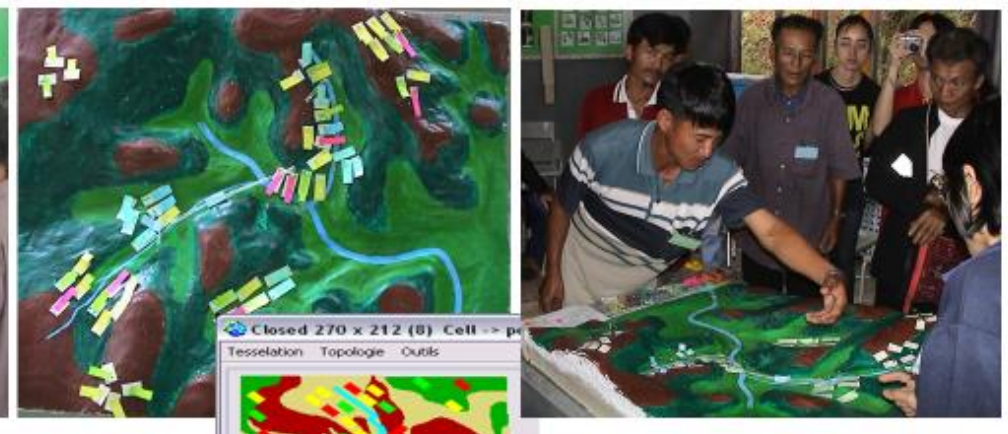

Avec les exploitations les plus aisées

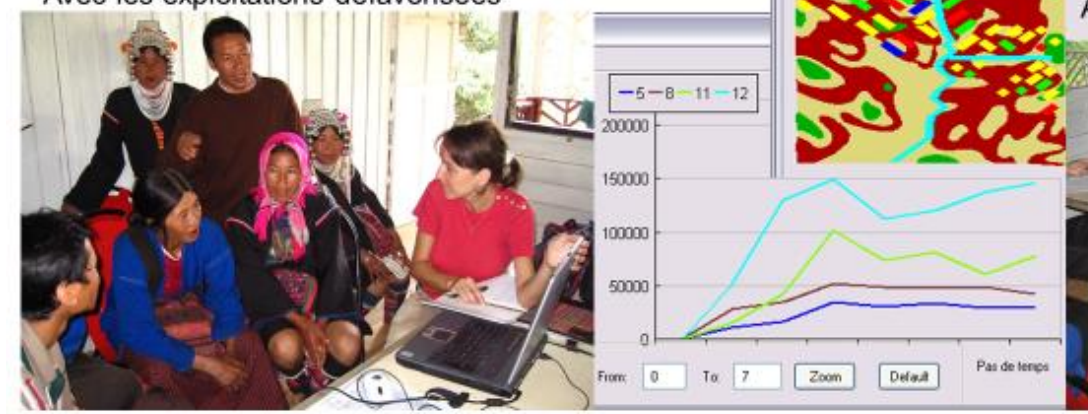

西

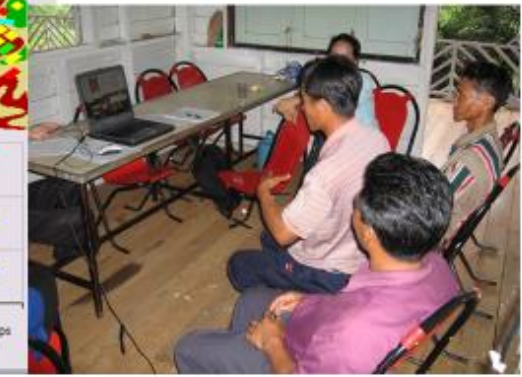

Figure 5: Simulations participatives des deux groupes d'agriculteurs s'affrontant sur le type d'infrastructure de stockage et les règles de partage de l'eau d'irrigation à mettre en œuvre

Après ce $3^{\text {ème }}$ atelier, les agriculteurs déposèrent une demande de financement de retenues collinaires auprès de l'administration du sous-district, mais sans succès. Ils souhaitaient aussi que de nouvelles simulations participatives puissent avoir lieu après leur construction porteuse d'une opportunité pour remettre en question les précédentes règles inégalitaires d'accès à l'eau. Une visite du bassin versant en 2013 montrait que 9 petites retenues collinaires avaient finalement pu être construites, avec le soutien financier du district, et que deux ateliers de conditionnement du thé vert fonctionnaient au village, un privé et un coopératif correspondant aux deux catégories d'exploitants qui s'étaient affrontés lors du dernier atelier. Bien que de nombreux autres facteurs aient influencé le cours des évènements, il est raisonnable de penser que l'intervention décrite cidessus contribua à ce que les acteurs locaux atteignent une capacité d'organisation et de négociation suffisante pour défendre et réaliser ces projets.

\section{Évaluation des effets de l'approche, des méthodes et outils mis en œuvre}

En complément d'un suivi-évaluation continu des effets des activités ComMod sur les participants par les animateurs, une évaluation externe fut réalisée par un binôme de sociologues indépendantes (van Paassen et Patamadit, 2007). Leur rapport abordait les points suivants : Quelles activités avaient le plus intéressé les participants et pourquoi ? Qu'avaient-ils appris sur les thèmes successivement examinés ainsi que sur les autres acteurs ? L'engagement collectif et le renforcement de leurs capacités, les nouvelles pratiques observées, les effets spécifiques des méthodes appliquées, l'ancrage des décisions dans le contexte et la reconnaissance du réseau d'acteurs afin d'obtenir les moyens de réaliser leur projet territorial.

\section{Apprentissage et prise de décision en gestion des ressources}

L'approche utilisée était bien plus positivement perçue par les agriculteurs participants que par leur encadrement habitué à fonctionner dans un cadre institutionnel hiérarchique descendant, et peu enclin à s'engager dans la conception ascendante d'un projet territorial les amenant à céder une partie de leur pouvoir local. Ainsi le vulgarisateur agricole limitait son rôle à l'organisation et l'observation des ateliers. Les agriculteurs appréciaient la capacité à représenter la dynamique de leur système agricole et la possibilité d'anticiper les conséquences négatives de certains 
comportements et pratiques. Malgré le faible niveau d'éducation formelle de la plupart, ils participaient activement aux dynamiques familières simulées, surtout lors des séances de JdR dont les composants et règles reposaient sur leur quotidien. Les agriculteurs découvraient d'autres façon de penser et discuter de thèmes non abordés dans la routine quotidienne. Pour plusieurs participants, les scénarios simulés donnaient l'idée de nouvelles pratiques (pour limiter l'érosion ou se partager l'eau d'irrigation) et ils consultaient ensuite leurs voisins sur les détails techniques avant de les tester. L'agriculteur leader religieux charismatique déclarait même que les scénarios simulés le stimulaient à penser bien au-delà des résultats visualisés ! Tandis que le vulgarisateur considérait qu'il y avait maintenant deux catégories d'agriculteurs dans le village selon qu'ils avaient ou pas participé à l'intervention. Il disait ne plus pouvoir travailler comme avant avec les participants aux activités décrites qui lui posaient maintenant des questions difficiles et allaient facilement chercher de l'information et des ressources ailleurs.

\section{Capacité à s'impliquer dans les décisions collectives}

Plusieurs agricultrices de type $A$, remarquaient que cette fois leurs avis comptaient à la façon dont les outils de simulation évoluaient d'un atelier à l'autre, les animateurs les laissant décider des inflexions de l'intervention que plusieurs autres villageois souhaitaient rejoindre car les débats durant les ateliers percolaient et touchaient d'autres ménages. Ainsi, selon les évaluateurs externes, des exploitantes de type $\mathrm{A}$ gagnèrent en confiance et se mirent à débattre des sujets examinés dans leurs groupes d'entraide. Les participants réclamèrent de nouveaux ateliers de simulation participative impliquant plus de villageois, ce qui ne put avoir lieu avant la fin du projet. Les participants déclaraient avoir appris des dynamiques agricoles simulées. Les apprentissages individuels observés et l'amélioration de la capacité d'organisation collective étaient notables suite à la prise de conscience de l'urgence du changement de pratiques afin d'éviter l'éviction de ce site. Mais l'intervention n'avait pas réussi à influencer le mode de prise de décision au niveau du sousdistrict où le discours favorable à l'élaboration ascendante de projets, dans le cadre de la politique nationale décentralisatrice, ne se traduisait pas par l'allocation de ressources pour les réaliser. Plusieurs années de démarches furent nécessaires afin de les obtenir d'autres sources (au niveau du district et de l'association provinciale de soutien à l'ethnie Akha). L'implication tardive d'administrateurs territoriaux dans la dernière séquence fut insuffisante pour améliorer la légitimité du collectif et de son projet. Le fait que le projet d'aménagement hydraulique soit encore débattu dans le village longtemps après le dernier atelier faisait apparaître cette dernière séquence comme la plus marquante pour les évaluateurs. Les séquences successives avaient initié les participants à une manière alternative de construire un projet, mais cette façon de faire se heurtait au fonctionnement institutionnel de l'administration locale.

\section{Production de nouveaux savoirs}

Elle eut lieu au niveau des pratiques individuelles (vis-à-vis du risque d'érosion, de l'accès aux sources de crédit pour investir, du partage de l'eau agricole) et collectives (intérêts de l'allongement de la durée des prêts pour l'accès des exploitations $A$ et $B$ aux plantations, gestion plus équitable de l'irrigation par des retenues collinaires distribuées). Les participants montraient aussi une meilleure compréhension des processus complexes liés aux trois thèmes successivement examinés. Dès 2005 , le leader religieux charismatique du village, qui colportait les débats dans d'autres villages, déclarait « je ne peux pas m'adresser de la même manière à ceux qui ont participé aux activités et aux autres à cause de leur différence de compréhension des problèmes ».

Dès la première séquence ComMod, la création d'un espace d'échanges intensifs et de compréhension mutuelle des différents points de vue, sans esquiver les divergences (cf. la $3^{\text {ème }}$ séquence), facilitait la conduite des activités de diagnostic complémentaires à l'enquête initiale entre chacune des séquences. Cela se traduisait par l'obtention de données et connaissances de qualité sur des sous-systèmes clés (pratiques illégales d'articulation du crédit formel et informel, règles limitant l'accès à l'irrigation, etc.). 


\section{Modification des perceptions des autres acteurs et apprentissage social}

Les ateliers procuraient un espace innovant pour prendre du recul par rapport au quotidien et partager des opinions dans une ambiance non menaçante. Bien que les participants soient du même village, plusieurs mentionnaient avoir découvert des stratégies, modes de prise de décision et pratiques chez leurs voisins, particulièrement lors des séances de JdR de l'ultime séquence.

L'élargissement des réseaux d'interactions sociales était constaté, mais ils restaient souvent limités au village et insuffisants pour accéder aux ressources nécessaires afin de réaliser le projet d'aménagement, l'équipe de recherche devait assurer le lien entre les villageois et les responsables administratifs et politiques locaux.

\section{Changement de pratiques}

L'évaluation mentionna des effets indirects non attendus. Plusieurs agricultrices de type A s'engageaient dans la lutte antiérosive en construisant de fossés déclinants pour diminuer les longueurs de pentes et dévier ruissellement et sédiments transportés vers des jachères. Sans attendre la réalisation d'un aménagement, un participant de type $C$ organisa le partage de l'eau d'irrigation avec quelques voisins le long d'un cours d'eau et conseilla d'autres agriculteurs intéressés. D’autres pratiques, que les acteurs reliaient aux activités décrites, étaient éloignées des thèmes traités en ateliers où elles émergèrent, comme la fertilisation et le sarclage des vergers.

\section{Effets spécifiques des méthodes et outils}

Les acteurs locaux appréciaient le maniement de modèles simplifiant la complexité des dynamiques examinées mais faisant sens à leurs yeux. Ils s'imposaient parfois des contraintes supplémentaires, non exigées par les règles du JdR, simplement parce qu'ils y étaient confrontés dans la réalité.

Les séances de JdR étaient l'activité la plus appréciée des participants. Avec le recul pris sur la réalité, cet outil permettait de libérer la parole, d'apprendre en observant les actes des autres et d'interagir dans une atmosphère non contrainte. Le jeu était pris au sérieux et tricher n'était pas possible sans être rappelé à l'ordre ! Les joueurs appréciaient aussi le fait que les conséquences des actions soient visibles sur le plateau ou à l'écran sans avoir à défier leurs responsables du regard. Les phases de débat en séance plénière après les simulations étaient appréciées car elles créaient un espace de communication permettant l'expression nuancée des différents points de vue et étaient modérées par des animateurs/trices neutres. Au terme de la séquence finale, le débat entre les deux options pour la gestion de l'eau agricole se poursuivit durant plusieurs semaines et s'élargit à l'ensemble des villageois.

Après avoir participé aux séances de JdR, les agriculteurs n'avaient pas de difficultés majeures à suivre les simulations informatiques de scénarios et se disaient stimulés à agir après avoir découvert les conséquences possibles des pratiques testées. Mais ces simulations participatives entraînèrent moins de débats animés et de réflexions critiques que les séances de JdR. Sauf lors des simulations en sous-groupes plus homogènes de la dernière séquence permettant de gérer plus équitablement l'expression de chacun. Cette étape favorisa la mise en confiance des acteurs les moins influents avant le débat en séance plénière.

\section{Points forts et faiblesses de l'approche}

L'approche et les outils mobilisés ont permis la transformation d'un problème de dégradation des terres, initialement abordé aux échelles de la parcelle cultivée avec un focus négatif sur les pertes de terre, en un projet territorial impliquant de profonds changements dans la combinaison des cultures sur les exploitations ainsi que leur mode de gestion (du pluvial à l'irrigué). L'approche a permis de prendre en compte l'incertitude croissante mais aussi les opportunités de la situation territoriale, la rapidité des changements en cours, ainsi que les différences de valeurs et intérêts entre types d'agriculteurs. Elle leur a permis d'explorer des directions innovantes pour solutionner le problème initial, en contestant la pertinence des connaissances mobilisées jusque-là et des 
politiques locales menées pour lutter contre l'érosion.

\section{Complémentarité entre diagnostic et modélisation d'accompagnement}

Ces travaux transdisciplinaires ont produit des connaissances sur plusieurs questions clés bien définies en passant les frontières entre sciences et savoirs empiriques ou experts. Les agronomes impliqués ont contribué à gérer un problème agri-environnemental en s'engageant dans des interactions sociales suivies avec les acteurs du territoire concernés pour améliorer la prise de décision collective et faire émerger un engagement négocié dans l'action.

Les activités ComMod ont pu être initiées grâce à l'enquête-diagnostic préliminaire qui avait permis de sceller le partenariat entre les intervenants extérieurs et les acteurs locaux. La figure 6 montre comment les activités d'enquête diagnostic complémentaires menées entre les séquences successives ont servi à adapter (sans les complexifier) les modèles multi-agent implémentés en JdR et simulateurs informatiques associés utilisés pour explorer des scénarios identifiés lors des parties jouées et évalués avec les indicateurs intéressant les agriculteurs.

Initiée à partir d'un modèle complexe d'accompagnement du chercheur intégrant les résultats du diagnostic initial, l'intervention se poursuivait au moyen d'une série de modèles d'accompagnement des acteurs locaux dans leurs apprentissages. Enracinés dans leurs préoccupations majeures et leur vécu, ils se les appropriaient grâce à l'outil JdR et leur intérêt et engagement à poursuivre l'apprentissage était entretenu. Cette famille de modèles simples, et leurs implémentations en JdR et leurs simulateurs informatiques associés, constituaient autant de jalons du cheminement accompli avec ce collectif. Afin d'assurer leur pertinence et utilité, l'adaptation continue des modèles aux questionnements successifs soulevés nécessite une grande flexibilité de la part du formalisme ouvert choisi (les systèmes multi-agent ici), mais surtout une grande réactivité de la part des modélisateurs de l'équipe d'animation.

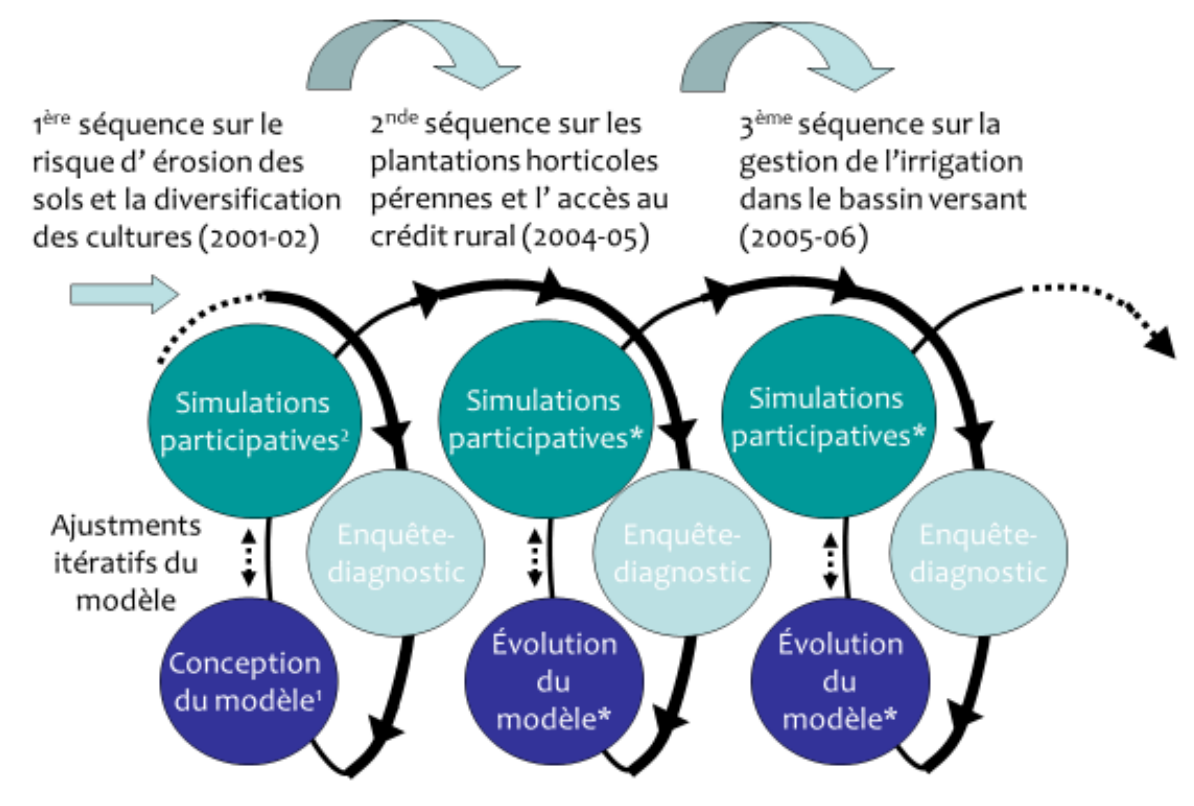

' Modèle conceptuel, ${ }^{2}$ Implémentations en jeu de rôles \& simulateur informatique associés

Figure 6 : Articulation entre enquête-diagnostic et modélisation d'accompagnement au fil de l'étude de cas. Source : adapté de Barnaud et al., 2008b

La nécessaire institutionnalisation de l'approche

La négociation animée sur l'irrigation entre les types d'agriculteurs ne put aboutir avant l'arrêt de son accompagnement par un projet limité dans le temps, sans qu'un organisme de développement et un animateur local puisse prendre le relais et poursuivre la négociation avec les autorités locales. 
Il aurait sans doute fallu entretenir un dialogue plus précoce et suivi avec les échelons administratifs et politiques supra-villageois afin d'éviter l'échec de leur intégration tardive dans l'intervention seulement quand les agriculteurs se sentirent prêts à interagir avec eux. Sauf cas de passage rapide à l'action collective, quand la gouvernance administrative et politique locale est réellement engagée dans ce type d'approche ascendante, ce cas montre la nécessité de son institutionnalisation afin de pouvoir accompagner les actions concrètes entreprises sur le terrain et réaliser un suivi-évaluation à moyen terme. Associer d'emblée l'étude de la gouvernance locale des ressources renouvelables aux activités de modélisation d'accompagnement est une voie à explorer pour aller dans ce sens et faciliter l'extrapolation ultérieure des résultats. Cela permettrait aussi d'aider l'animateur ComMod à gérer le dilemme de la posture à adopter en cas de blocage entre intervenir ou observer comment les relations sociales évoluent et ouvrent (ou pas) la voie aux changements (Barnaud, 2008).

Une telle institutionnalisation permettrait également de mieux valoriser les nombreux résultats issus de l'enquête-diagnostic initiale. Elle avait notamment permis de mettre en évidence l'efficacité de plusieurs pratiques paysannes antiérosives adaptées aux systèmes de culture annuelle pratiqués (andains filtrants d'adventices en courbe de niveau lors des sarclages, fossés déclinants écourtant les longueurs de pente), jusque-là ignorées des projets régionaux et inégalement connues selon les villages.

\section{Conclusion}

L'approche mise en œuvre sur ce terrain permit de multiplier les allers-retours entre l'enquêtediagnostic agronomique, la modélisation au laboratoire et plusieurs temps forts collectifs lors d'ateliers de simulations participatives au village. Leur rythme fut ralenti à cause notamment des exigences de calendrier et des activités de formation (notamment aux outils mobilisés) des étudiants impliqués. Dans un contexte de transformations agraires rapides, ce cas illustre une façon de combiner la longue enquête-diagnostic initiale et ses compléments ultérieurs avec l'usage de ses résultats en modélisation et simulation participative. Ceci afin de mieux prendre en compte le temps et l'incertitude dans les travaux d'agronomes en milieu paysan et d'offrir des éléments de réponse aux questions clés soulevées par les acteurs à la vitesse du changement de leur territoire. Ce cas montre aussi comment l'agronomie territoriale amène à changer la façon de poser le problème à résoudre et incite à l'adoption d'une posture transdisciplinaire et interactive.

Les effets sur les participants comprenaient différents types d'apprentissages individuels et l'amélioration de la capacité à communiquer et à participer à la gestion des ressources communes, notamment chez les types d'agricultrices/teurs les plus défavorisés. L'élargissement de leurs réseaux d'interactions sociales était significatif et lié à des changements de pratiques agricoles. Malgré le contexte institutionnel et culturel peu porteur, l'intensification des interactions et la participation à des débats collectifs étaient notables. Parmi les outils mobilisés, les séances de JdR et les débats en plénière sur les résultats des simulations étaient les plus appréciés.

Dans le contexte thaï, au potentiel de la situation administrative locale limité, il était nécessaire de travailler à long terme en formant de jeunes collègues à ce type d'approche nécessitant des compétences variées. Quatre jeunes chercheurs réalisèrent leur recherche doctorale dans ce domaine dans chacune des principales régions du pays où ils occupent des postes d'enseignantschercheurs universitaires et encadrent de nouveaux travaux dans ce domaine. Un JdR générique (WADIGA, pour "water distribution game», voir sa présentation à l'adresse: https://www.commod.org/modeles), dérivé de celui construit pour l'ultime séquence ComMod décrite, est utilisé lors de formations agronomiques en France, ainsi que dans d'autres écorégions aux dynamiques agraires similaires. 


\section{Remerciements}

Les auteurs remercient l'ensemble des partenaires des universités thaïlandaises de Chiang Maï et Chulalongkorn sans qui ces travaux n'auraient pu être réalisés. Ils remercient aussi l'Institut international de recherche sur le riz (IRRI), le "Challenge Program on Water and Food" (CPWF) du GCRAI, l'université Paris Nanterre, ainsi que l'initiative IT\&C de l'Union Européenne pour leurs soutiens financiers.

\section{Références citées}

Barnaud, C., 2008. Équité, jeux de pouvoir et légitimité : les dilemmes d'une gestion concertée des ressources renouvelables. Thèse de doctorat en géographie, Université Paris X Nanterre. 408p.

Barnaud, C., Promburom, T., Trébuil G., Bousquet F., 2007. An evolving simulation and gaming process to facilitate adaptive watershed management in mountain northern Thailand. Simulation and Gaming, 38: 398-420. Doi: 10.1177/1046878107300670

Barnaud, C., Bousquet, F., Trébuil, G., 2008a. Multi-agent simulations to explore rules for rural credit in a highland farming community of Northern Thailand. Ecological Economics, 66(4): 615-627. https://doi.org/10.1016/j.ecolecon.2007.10.022

Barnaud, C., Trébuil, G., Promburom, P., Bousquet F., 2008b. La modélisation d'accompagnement pour une gestion concertée des ressources renouvelables en Thaïlande. Économie rurale, 303-305: 39-59. https://economierurale.revues.org/512

Barnaud, C., van Paassen, A., Trebuil, G., Promburom, T., Bousquet, F., 2010. Dealing with Power Games in a Companion Modelling Process: Lessons from Community Water Management in Thailand Highlands. The Journal of Agricultural and Extension Education, 16(1): 55-74.

Boiffin, J., Papy, F., Eimberck, M., 1988. Influence des systèmes de culture sur les risques d'érosion par ruissellement concentré. I : Analyse des conditions de déclenchement de l'érosion. Agronomie, 8(8) : 663-673.

Boiffin, J., Benoît, M., Le Bail, M., Papy, F., Stengel, P., 2014. Agronomie, espace, territoire : travailler « pour et sur » le développement territorial, un enjeu pour l'agronomie. Cahiers Agricultures, 23 : 72-83. doi : 10.1684/agr.2014.0688

Bousquet, F., 2001. Modélisation d'accompagnement Simulations multi-agents et gestion des ressources naturelles et renouvelables. Mémoire d'HDR, Humanités et Sciences Sociales, Université Claude Bernard, Lyon I. 7op.

Etienne, M. (coord), 2010. La modélisation d'accompagnement : une démarche en appui au développement durable. Versailles : éditions Quae, collection Update Sciences \& Technologies.

Scott, J.C., 2013. Zomia ou l'art de ne pas être gouverné : une histoire anarchiste des hautes terres d'Asie du Sud-Est. Editions Seuil, essais. 768p.

Trébuil, G., Kam, S.P., Turkelboom, F., Shinawatra, B., 1997. Systems Diagnoses at Field, Farm and Watershed Levels in Diversifying Upland Agroecosystems: Towards Comprehensive Solutions to Farmers' Problems. In: Applications of systems approaches at the farm and regional levels. Vol.1. Teng P.S., Kropff M.J., Ten Berge H.F.M., Dent J.B., Lansigan F.P., Van Laar H.V. (eds). IRRI. Dordrecht: Kluwer Academic Publishers, 99-114.

Trébuil, G., Bousquet, F., Ekasingh, B., Baron, C., Le Page, C., 2005. A multi-agent model linked to a GIS to explore the relationship between crop diversification and land degradation in northern Thailand highlands. In: Bousquet F., Trébuil G., Hardy B. (eds). Companion Modeling and Multi-Agent Systems for Integrated Natural Resource Management in Asia. Cirad \& International Rice Research Institute, Los Baños, Laguna, Philippines. 167-190. 
Trébuil, G., Ekasingh, B., Ekasingh, M., 2006. Agricultural Commercialisation, Diversification, and Conservation of Renewable Resources in Northern Thailand Highlands. Moussons, 9-10: 131-155. https://dx.doi.org/10.4000/moussons.2005

Turkelboom, F., Trébuil, G., Vejpas C., 1996. Starting from the farmers' fields: On-farm Analysis and Development of conservation strategies on steeplands. In: Sombatpanit S., Zobisch M. A., Sanders D. W., Cook M. G. (eds). Soil Conservation Extension: from Concepts to Adoption. Soil and Water Conservation Society of Thailand, Bangkok, Thailand. 141-152.

Turkelboom, F., Trébuil, G., 1998. A Multiscale Approach for On-farm Erosion Research: Application to Northern Thailand Highlands. In: Penning de Vries, F.W.T, Fahmuddin Agus, Kerr J. (eds). Soil Erosion at Multiple Scales: Principles and Methods for Assessing Causes and Impacts. CABI and IBSRAM. 51-71.

Turkelboom, F., 1999. On-farm diagnosis of steepland erosion in northern Thailand - Integrating spatial scales with household strategies. Doctorate thesis, K.U. Leuven University, Belgium. 309p.

Turkelboom, F., Poesen, J., Trébuil, G., 2008. The multiple land degradation effects caused by landuse intensification in tropical steeplands: A catchment study from northern Thailand. CATENA, 75: 102-116. Doi: 10.1016/j.catena.2008.04.012

van Paassen, A., Patamadit, I., 2007. External evaluation of the Mae Salaep Commod case study in Chiang Rai province, Northern Thailand- Final report. Projet "La modélisation d'accompagnement : une pratique de recherche en appui au développement durable", programme fédérateur “Agriculture et Développement Durable”, ANR, 42p. 